\title{
Initial experience with a microprocessor controlled current based defibrillator
}

\author{
G W N DALZELL, S R CUNNINGHAM, J ANDERSON, * A A J ADGEY \\ From the ${ }^{\star}$ University of Ulster, Jordanstown, and Regional Medical Cardiology Centre, Royal Victoria \\ Hospital, Belfast, Northern Ireland
}

SUMMARY Intramyocardial current flow is a critical factor in successful ventricular defibrillation. The main determinants of intramyocardial current flow during transthoracic countershock are the selected energy and the transthoracic impedance of the patient. To optimise the success of the first shock and to titrate energy dosage according to each patient's transthoracic impedance, a microprocessor controlled current based defibrillator was developed. It was compared with a conventional energy based protocol of $200 \mathrm{~J}$ (delivered energy), $200 \mathrm{~J}$, then $360 \mathrm{~J}$ if required in 42 consecutive episodes of ventricular fibrillation in 33 men and seven women. The mean (SD) predicted transthoracic impedance was 69.9 (14.0) $\Omega$. First shock success with the standard protocol was $80.9 \%$, and first or second shock success was $95.2 \%$. The microprocessor controlled current based defibrillator automatically measured transthoracic impedance and calculated the energy required to develop a selected current in each patient. A current protocol of $30 \mathrm{~A}, 30 \mathrm{~A}$, then $40 \mathrm{~A}$, if required, was used in 29 men and 12 women with 41 episodes of ventricular fibrillation. Transthoracic impedance (mean 65.1 (15.9) $\Omega$ ) was similar to that in the energy protocol group and success rates for first shock $(82.9 \%)$ and first or second shocks $(97.5 \%)$ were also similar. The mean delivered energy per shock with the current based defibrillator for first or second shock success was significantly less $(144.8 \mathrm{~J})$ with the energy protocol $(200 \mathrm{~J})$. The mean peak current of successful shocks was also significantly reduced $(29.0 v 31.9 \mathrm{~A})$.

A current based defibrillator titrates energy according to transthoracic impedance; it has a success rate comparable to conventional defibrillators but it delivers significantly less energy and current per shock.

Ventricular defibrillation is successful when a critical mass of myocardium is depolarised. ${ }^{1}$ Although conventional defibrillators are calibrated in terms of energy, it has been suggested that current rather than energy is more important in correcting ventricular fibrillation. ${ }^{23}$ Electrical countershock can be associated with adverse effects and ideally ventricular fibrillation should be terminated with a threshold shock. However, in humans it is not possible to determine the electrical threshold for transthoracic ventricular defibrillation. Nevertheless, the principle of giving the smallest effective electrical shock remains valid. The concept of advance prediction of transthoracic impedance ${ }^{4}$ before defibrillator dis-

Requests for reprints to Dr A A J Adgey, Regional Medical Cardiology Centre, Royal Victoria Hospital, Belfast BT12 6BA, Northern Ireland.

Accepted for publication 2 February 1989 charge suggested that it might be possible to develop a defibrillator that delivers a fixed current. We have developed a microprocessor controlled current based defibrillator and in this study we compared it with a standard energy based defibrillator.

\section{Patients and methods}

We studied two groups of consecutive patients who had ventricular fibrillation within or outside hospital. In all patients pre-gelled adhesive electrocardiographic defibrillator pads $(12 \mathrm{~cm}$ diameter) were applied in the anteroapical position.

For the first $\mathbf{4 2}$ consecutive episodes of ventricular fibrillation in 40 (33 men, seven women) patients (mean age 61.5 years) we used a standard energy protocol of $200 \mathrm{~J}$ (delivered energy) for a first shock, followed by $200 \mathrm{~J}$ for a second shock if required and subsequent shocks of $360 \mathrm{~J}$ if necessary. Trans- 


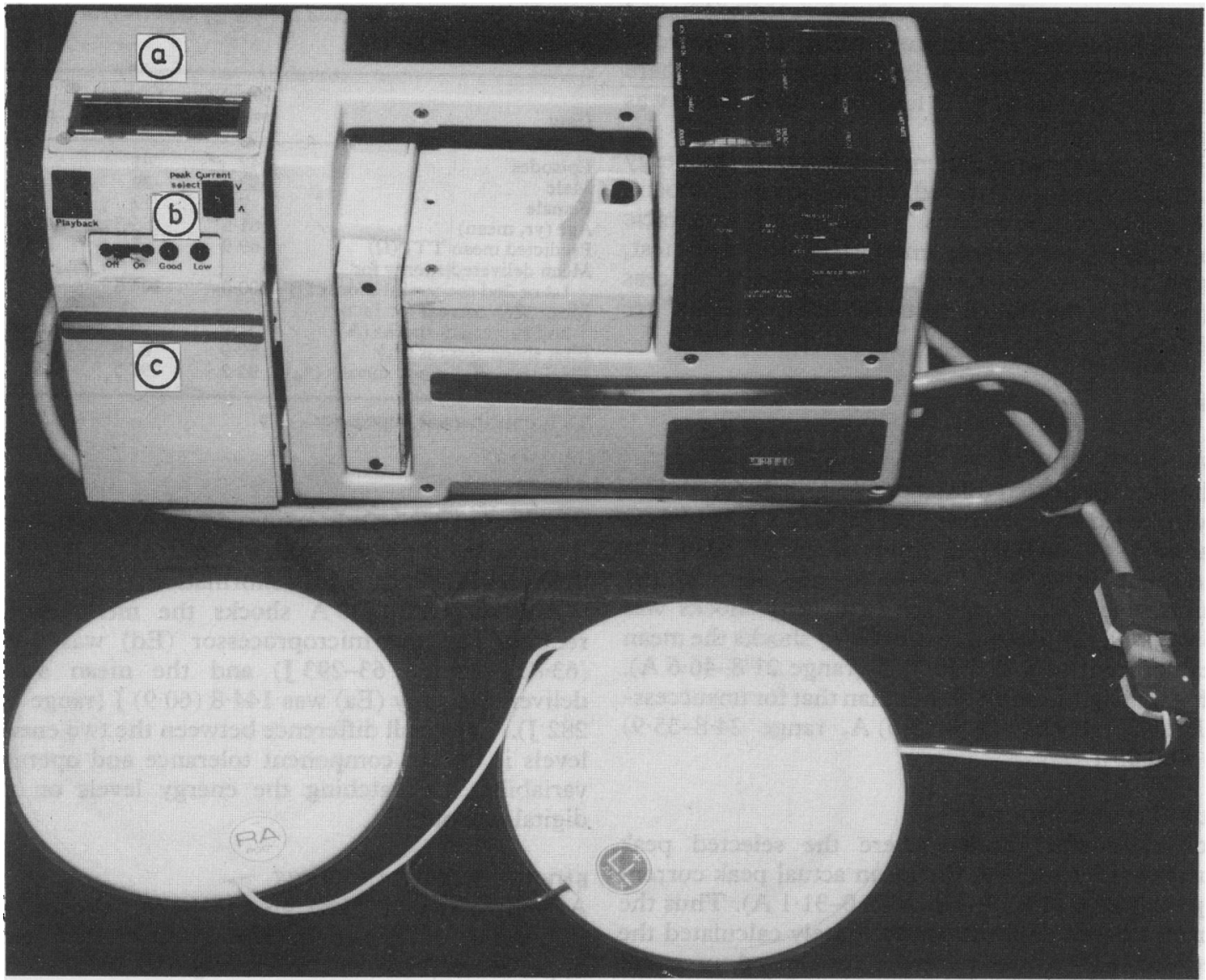

Fig 1 Microprocessor based current defibrillator. The microprocessor and device for measuring transthoracic impedance is mounted behind a standard liteguard 6 defibrillator. (a) Digital display; (b) thumbwheel switch; (c) microprocessor.

thoracic impedance was recorded immediately before defibrillator discharge by a $30 \mathrm{kHz}$ low amplitude $(100 \mu \mathrm{A})$ current applied via the electrode pads. We have previously shown that this method accurately predicts the actual transthoracic impedance to a defibrillatory shock, both in patients with atrial arrhythmias, ${ }^{5}$ and ventricular fibrillation. ${ }^{6}$ The peak transthoracic current generated by each discharge was also recorded for each shock.

In the next 41 consecutive patients with 41 episodes of ventricular fibrillation (29 men and 12 women, mean age 63.3 years) we used a microprocessor controlled current based defibrillator (fig 1). In a previous study that we performed in patients with ventricular fibrillation who were defibrillated by an energy protocol the mean peak current for successful defibrillation was $29 \cdot 2 \mathrm{~A}$. ${ }^{6}$ So we decided to use $30 \mathrm{~A}$ for the initial shock in this study. If this was unsuccessful we used a second $30 \mathrm{~A}$ shock. Sub- sequent shocks were to be $40 \mathrm{~A}$. The peak current (Is) was selected by means of a thumbwheel switch. The transthoracic impedance was automatically measured by a low amplitude high frequency current method described above. The microprocessor used the current selected, the predicted transthoracic impedance measurement, and pre-programmed defibrillator characteristics to "look-up" the energy required (Ed) to develop the current selected (Is). This energy level was digitally displayed. The defibrillator was then charged by the operator pressing the charge button and matching up the two energies that were displayed side by side on the digital display. This process occurred almost as soon as the charge button was pressed. There was good correlation between the energy required and delivered energy for each patient $(r=0.98)$

For each shock, the predicted transthoracic impedance, the required energy (Ed), the actual 
delivered energy (Ea), selected peak current (Is), and actual peak transthoracic current (Ip) were recorded in non-volatile memory and played back where required. The success or failure of each shock was recorded.

We then compared patients treated with the energy protocol with those treated by the current protocol. We analysed continuous variables (transthoracic impedance, peak current, energy) by Student's $t$ test, and discrete variables such as defibrillation success by $\chi^{2}$ and the fourfold table tests. Results are expressed as mean (1 SD).

\section{Results}

ENERGY PROTOCOL

The mean predicted transthoracic impedance for this group for first or second shocks was $69.9(14.0) \Omega$ (range 41.0-100.0 $\Omega$ ). A single shock of $200 \mathrm{~J}$ was successful in $80.9 \%(34 / 42)$ of episodes and the cumulative success rate for two $200 \mathrm{~J}$ shocks was $95 \cdot 2 \%$ (40/42). For successful $200 \mathrm{~J}$ shocks the mean peak current was $31.9(5 \cdot 7)$ A (range 21.8-46.6 A). This was significantly higher than that for unsuccessful $200 \mathrm{~J}$ shocks (28.4 (3.6) A, range 24.8-35.9) $(\mathrm{p}<0.05)$.

\section{CURRENT PROTOCOL}

For successful shocks where the selected peak current (Is) was $30 \mathrm{~A}$ the mean actual peak current (Ip) was 29.0 (1.4) A (range 25.0-31.1 A). Thus the current based defibrillator accurately calculated the required energy to develop the preselected current of $30 \mathrm{~A}$. This system accurately generated a constant current over a wide range of transthoracic impedances (fig 2).

The mean predicted transthoracic impedance of this group for first or second shocks was $65 \cdot 1(15 \cdot 9) \Omega$ (range 38.0-97.0 $\Omega$ ). A single shock of $30 \mathrm{~A}$ was successful in $82.9 \%(34 / 41)$ of episodes and the cumulative success rate for two $30 \mathrm{~A}$ shocks was

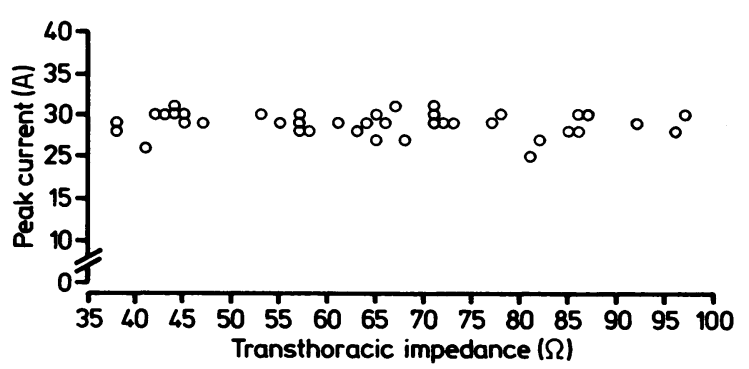

Fig 2 Graph of peak current versus predicted transthoracic impedance for all $30 \mathrm{~A}$ shocks (48 shocks, eight overlapping). A current based defibrillator can deliver a constant current over a wide range of impedances.
Table Comparison between the energy and current based protocols

\begin{tabular}{|c|c|c|c|}
\hline Data & $\begin{array}{l}\text { Energy } \\
\text { protocol }\end{array}$ & $\begin{array}{l}\text { Current } \\
\text { protocol }\end{array}$ & $p$ \\
\hline $\begin{array}{l}\text { Episodes } \\
\text { Male } \\
\text { Female } \\
\text { Age }(\text { yr, mean) } \\
\text { Predicted mean TTI }(\Omega) \\
\text { Mean delivered energy for }\end{array}$ & $\begin{array}{c}42 \\
33 \\
7 \\
61 \cdot 5 \\
69 \cdot 9\end{array}$ & $\begin{array}{l}41 \\
29 \\
12 \\
63 \cdot 3 \\
65 \cdot 1\end{array}$ & NS \\
\hline 1st or 2nd successful shocks (J) & $200 \cdot 0$ & $144 \cdot 8$ & $<0.001$ \\
\hline $\begin{array}{l}\text { 2nd successful shocks (A) } \\
\text { First shock success (\%) } \\
\text { First or second shock success (\%) }\end{array}$ & $\begin{array}{l}31 \cdot 9 \\
80 \cdot 9 \\
95 \cdot 2\end{array}$ & $\begin{array}{l}29 \cdot 0 \\
82 \cdot 9 \\
97 \cdot 5\end{array}$ & $\begin{array}{l}<0.05 \\
\text { NS } \\
\text { NS }\end{array}$ \\
\hline
\end{tabular}

TTI, transthoracic impedance.

$97.5 \%$ (40/41). Only one patient (transthoracic impedance $82 \Omega$ ) required a shock of $40 \mathrm{~A}(311 \mathrm{~J}$ 응 delivered) for successful defibrillation.

For successful $30 \mathrm{~A}$ shocks the mean energy $\subseteq$ required by the microprocessor $(\mathrm{Ed})$ was $148.8 \stackrel{\mathrm{D}}{\mathrm{D}}$ (63.8) J (range 63-293 J) and the mean actual $\overrightarrow{0}$ delivered energy (Ea) was $144.8(60.9) \mathrm{J}$ (range 61- 8 $282 \mathrm{~J}$ ). The small difference between the two energy levels is due to component tolerance and operator variability in matching the energy levels on the digital readout.

\section{ENERGY VERSUS CURRENT}

A comparison of these groups (table) showed no $\overrightarrow{\overrightarrow{0}}$ significant difference in the mean transthoracic 3 impedance. There was also no significant difference in success rates between the groups for the first shock (energy $80.9 \%$ versus current $82.9 \%, p>0.05$ ) and $\vec{\odot}$

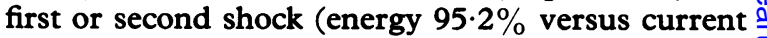
$97.5 \%, p>0.05$ ) (table). For first or second success- $\frac{\vec{\sigma}}{\vec{\sigma}}$ ful shocks, however, the mean delivered energy per shock with a current protocol was $144.8(60.9) \mathrm{J}$, ᄋ which was significantly less than the $200 \mathrm{~J}$ used for the conventional energy protocol $(p<0.001)$. Also o the mean peak current for first or second successful shocks with the current based protocol was 29.0 음 (1.4) A, significantly less than the mean of $31.9 \mathrm{~N}$ (5.7) A administered by the energy protocol or $(\mathrm{p}<0.05)$.

\section{Discussion}

DC defibrillation remains a cornerstone of modern cardiology, and since its widespread introduction in $\stackrel{?}{?}$ the 1960s it has saved thousands of lives. Neverthe- $\square$ less the potentially adverse effects of electrical countershock must not be ignored. It has been shown in animal experiments that microscopic and macro- $\mathbb{D}$ scopic damage are produced in myocardial tissue 
after countershock. ${ }^{3}$ The ultrastructural changes include alterations in membrane permeability with resultant accumulation of intracellular calcium and areas of contraction band formation. ${ }^{78}$ High shock intensities may produce secondary shock induced membrane depolarisation and hence refibrillation of the ventricle. 9 Also, high energy shocks cause more cardiac damage than low energy shocks. ${ }^{10}$

These effects have been shown only in animal experiments and are subject to the criticism that massive overdoses of energy were generally used. There is only one reported case of macroscopic and microscopic damage induced by DC shock in man. ${ }^{11}$ It is not yet known if a single shock of $200 \mathrm{~J}$ or $360 \mathrm{~J}$ produces myocardial damage in man because of the practical difficulties of detecting such ultrastructural damage. Nevertheless, complete heart block after the correction of ventricular fibrillation in man was more common after $320 \mathrm{~J}$ shocks than after $175 \mathrm{~J}$ countershocks. ${ }^{12}$ The principle that it is best to use the lowest effective energy level remains valid.

It is likely that current rather than energy is more important in successful defibrillation. Successful defibrillation occurs when a significant intramyocardial current causes depolarisation of cells facing the cathode and hyperpolarisation of cells facing the anode. ${ }^{2}$ Current density is probably the critical determinant ${ }^{13}$ although there is no method of measuring this in man.

During defibrillation the peak transthoracic current generated is determined by the energy selected and by the transthoracic impedance of the patient. It is known that transthoracic impedance varies significantly between patients ${ }^{1415}$ and for this reason use of a fixed energy level will result in a correspondingly variable range of generated transthoracic currents. In this study we showed that a microprocessor based current defibrillator allows energy to be titrated according to the individual patient's transthoracic impedance. This avoids unnecessarily high energy shocks. Patients with a low transthoracic impedance require less energy to generate a peak transthoracic current of $30 \mathrm{~A}$ than patients with a high transthoracic impedance.

A current defibrillator that uses a current generator rather than a voltage generator has been developed. ${ }^{16}$ This device uses the principle of electromagnetic impulse conservation in an inductor charged with a pre-set current. This unit is cumbersome and it is not portable. Lerman et al also suggested a current based system for defibrillation and reported a similar reduction in energy and current for successful shocks delivered to patients undergoing electrophysiological testing. ${ }^{17}$ However, this system did not use a microprocessor, and the transthoracic impedance had to be measured in advance of the study with manual calculations before ventricular fibrillation was induced. Obviously such an approach could not be used in emergencies.

We have developed and confirmed the efficacy of a microprocessor controlled current based defibrillator. This system permits the rapid delivery of an accurately calibrated preselected transthoracic current to patients with ventricular fibrillation. Standard defibrillators can be easily modified to become current based systems with the addition of the microprocessor and facilities for measuring transthoracic impedance. This device is as effective as conventional defibrillators but it delivers significantly less energy and current per shock than commercially available energy based machines.

\section{References}

1 Zipes DP, Fischer J, King RM, Nicoll A deB, Jolly WW. Termination of ventricular fibrillation in dogs by depolarizing a critical amount of myocardium. Am J Cardiol 1975;36: 37-44.

2 Geddes LA, Tacker WA. Ventricular fibrillation and defibrillation. Australian Physical and Engineering Sciences in Medicine 1983;6:9-19.

3 Thomas ED, Ewy GA, Dahl CF, Ewy MD. Effectiveness of direct current defibrillation. Role of paddle electrode size. $\mathrm{Am}$ Heart J 1977;93:463-7.

4 Geddes LA, Tacker WA, Schoenlein W, Minton M, Grubbs S, Wilcox $P$. The prediction of the impedance of the thorax to defibrillating current. Med Instrum 1976;10:159-62.

5 Dalzell GWN, Magee H, Anderson J, Adgey AAJ. Predicted and actual impedance in cardioversion [Abstract]. Circulation 1986;74(suppl):388.

6 Cunningham SR, Magee H, Anderson J, Adgey AAJ. Patients in ventricular fibrillation-predicted and actual transthoracic impedance and peak current at defibrillation [Abstract]. $P A C E$ 1987;10:975.

7 Warner ED, Dahl C, Ewy GA. Myocardial injury from transthoracic defibrillator countershock. Arch Pathol 1975;99: 55-9.

8 Van Vleet JF, Tacker WA, Geddes LA, Ferrans VJ. Acute cardiac damage in dogs given multiple transthoracic shocks with a trapezoidal wave-form defibrillator. Am J Vet Res 1977; 38:617-26.

9 Jones JL, Jones RE. Postshock arrhythmias -2 possible cause of unsuccessful defibrillation. Crit Care Med 1980;8:167-71.

10 Patton JN, Allen JD, Pantridge JF. The effects of shock energy, propranolol, and verapamil on cardiac damage caused by transthoracic countershock. Circulation 1984;69:357-68.

11 Karch SB, Billingham ME. Morphologic effects of defibrillation: a preliminary report. Crit Care Med 1984;12:920-1.

12 Weaver WD, Cobb LA, Copass MK, Hallstrom AP. Ventricular defibrillation-a comparative trial using $175 \mathrm{~J}$ and $320 \mathrm{~J}$ shocks. N Engl J Med 1982;307:1101-6.

13 Hoyt R, Grayzel J, Kerber RE. Determinants of intracardiac current in defibrillation. Experimental studies in dogs. Circulation 1981;64:818-23.

14 Patton JN, Pantridge JF. Current required for ventricular defibrillation. Br Med J 1979;i:513-4.

15 Machin JW. Thoracic impedance of human subjects. Med Biol Eng Comput 1978;16:169-78.

16 Monzon JE, Guillen SG. Current defibrillator: new instrument of programmed current for research and clinical use. IEEE Trans Biomed Eng 1985;32:928-34.

17 Lerman BB, Haines DE, DiMarco JP, Wesley RC. An improved method for clinical defibrillation: current-based DC countershock [Abstract]. Circulation 1986;74(suppl II):389. 